\title{
Clinical phenotype features and genetic etiologies of 38 children with progressive myoclonic epilepsy
}

Jing Zhang ${ }^{1}$, Ying Yang ${ }^{1}$, Xueyang Niu', Jiaoyang Chen ${ }^{1}$, Wei Sun², Changhong Ding ${ }^{3}$, Lifang Dai ${ }^{3}$, Liping Zhang ${ }^{4}$, Qi Zeng ${ }^{1}$, Yi Chen ${ }^{1}$, Xiaojuan Tian ${ }^{1}$, Xiaoling Yang ${ }^{1}$, Taoyun Ji ${ }^{1}$, Zhixian Yang ${ }^{1}$, Yanling Yang ${ }^{1}$, Yuwu Jiang ${ }^{1}$ and Yuehua Zhang ${ }^{1 *}$

\begin{abstract}
Background: Progressive myoclonic epilepsy (PME) is a group of neurodegenerative diseases with genetic heterogeneity and phenotypic similarities, and many cases remain unknown of the genetic causes. This study is aim to summarize the clinical features and study the genetic causes of PME patients.

Methods: Sanger sequencing of the target gene, Next Generation Sequencing (NGS) panels of epilepsy, trio-based Whole Exome Sequencing (WES) and detection of cytosine-adenine-guanine (CAG) repeat number were used to investigate the genetic causes of PME patients.

Results: Thirty-eight children with PME whose seizure onset age ranged from 3 months to 12 years were collected from February 2012 to November 2019 in three hospitals in Beijing, China. The seizure types included myoclonic seizures ( $n=38)$, focal seizures $(n=19)$, generalized tonic-clonie seizure (GTCS) $(n=13)$, absence seizures $(n=4)$, atonic seizures $(n=3)$, epileptic spasms $(n=2)$ and tonic seizures $(n=1)$. Twenty-seven cases were sporadic and 11 had family members affected. Established PME-related genes were identified in 30 out of 38 (78.9\%) patients who had either recessively inherited or de novo heterozygous mutations. Among these 30 cases, there were 12 cases (31.6\%) of neuronal ceroid lipofuscinoses (the causing gene contains TPP1, PPT1, CLN5, CLN6 and MFSD8), two cases of sialidosis (the causing gene is NEU1), two cases of neuronopathic Gaucher disease (the causing gene is GBA), one case of spinal muscular atrophy-progressive myoclonic epilepsy (the causing gene is ASAH1), four cases of KCNC1 mutation-related PME, four cases of KCTD7 mutation-related PME, two cases of TBC1D24 mutation-related PME, one case of GOSR2 related PME, and two of dentatorubral-pallidoluysian atrophy (the causing gene is ATN1). In total, 13 PME genes were identified in our cohort. The etiology was not clear in eight patients.

Conclusion: PME is a group of clinically and genetically heterogeneous diseases. Genetic diagnosis was clear in 78.9\% of PME patients. Various of genetic testing methods could increase the rate of genetic diagnosis. Neuronal ceroid lipofuscinoses (NCL) is the most common etiology of PME in children. Nearly one third PME children were diagnosed with NCL. GOSR2 related PME was in our cohort in Asia for the first time.
\end{abstract}

Keywords: Progressive myoclonic epilepsy, Genotype, Phenotype

\footnotetext{
* Correspondence: zhangyhdr@126.com

'Department of Pediatrics, Peking University First Hospital, No. 1 of Xian Men

Street, Xicheng District, Beijing 100034, China

Full list of author information is available at the end of the article
}

(c) The Author(s). 2020 Open Access This article is licensed under a Creative Commons Attribution 4.0 International License, which permits use, sharing, adaptation, distribution and reproduction in any medium or format, as long as you give appropriate credit to the original author(s) and the source, provide a link to the Creative Commons licence, and indicate if changes were made. The images or other third party material in this article are included in the article's Creative Commons licence, unless indicated otherwise in a credit line to the material. If material is not included in the article's Creative Commons licence and your intended use is not permitted by statutory regulation or exceeds the permitted use, you will need to obtain permission directly from the copyright holder. To view a copy of this licence, visit http://creativecommons.org/licenses/by/4.0/. 


\section{Background}

Progressive myoclonic epilepsy (PME) is a group of regressive neurologic diseases. The clinical features of PME include myoclonus, multiple seizure types, progressive neurological regression, and cerebral and/or cerebellar atrophy $[1,2]$. Some PME cases had distinguishing biomarkers, such as lysosomal enzyme tripeptidyl peptidase (TPP1) enzyme or lysosomal palmitoyl protein thioesterase (PPT1) for neuronal ceroid lipofuscinoses (NCL) and characteristic clinical feature, such as cherry red spot in the maculae for Sialidosis, which could help us to make accurate disease diagnosis and to choose appropriate genetic tests. However, in most cases, there are a lack of distinguishing clinical features or biomarkers. Consequently, many cases remain unknown of the genetic causes. With the clinical application of the next generation sequencing (NGS), more and more PME related genes were identified [3-8]. We have summarized the genetic and clinical features of 26 PME patients in preliminary work [9].

This study is aim to summarize the clinical features of 38 children who were diagnosed with PME, and to identify the causes by choosing appropriate genetic testing methods. The PME patients were collected from three hospitals (Peking University First Hospital, Xuanwu Hospital and Beijing Children's Hospital) in Beijing China from February 2012 to November 2019.

\section{Materials and methods}

PME is characterised by myoclonic seizures, tonic-clonic seizures, and progressive neurological deterioration, typically with cerebellar signs and dementia [10]. The patients included in this study met the following criteria [11]: (1) Myoclonic seizures, with or without generalized convulsive seizures; (2) Mental and/or motor development delay or regression, and the patients could have or not have the features as (3) Cerebellar ataxia; (4) Cerebral and/or cerebellar atrophy.

The clinical data and peripheral blood DNA from children with PME and their parents and other family members were collected. This study was approved by the Ethics Committee of Peking University First Hospital. Parental written informed consent was obtained for all children enrolled in this study.

Sanger sequencing of the target genes was conducted in children who had specific clinical feature or biochemical results. For example, the sanger sequencing for TPP1 would be done when tripeptidyl peptidase 1 activity deficiency was found, NEU1 would be tested when cherry red spot in the maculae was observed, and GBA would be tested when deficiency of the lysosomal enzyme, glucocerebrosidase was found. NGS panels of epilepsy and triobased Whole Exome Sequencing (WES) were performed in children without distinguish biomarkers. Two families had clinical features of Dentatorubral-pallidoluysian atrophy (DRPLA), then the probands and affected family members received the detection of cytosine-adenineguanine (CAG) repeat number.

\section{Results}

Thirty-eight patients diagnosed with PME were enrolled in this study. In the 38 patients, the onset symptoms were seizures in 30 patients, mental and/or motor developmental regression in seven patients, and thrombocytopenia and spleen enlarged in one patient. The seizure onset age ranged from 3 months to 12 years. Seizures were captured in 20 patients during electroencephalogram (EEG) recording, which including myoclonic seizures $(n=16)$, focal seizures $(n=3)$, GTCS $(n=1)$, absence seizures $(n=3)$, atonic seizures $(n=2)$, epileptic spasm $(n=2)$ and tonic seizure $(n=1)$. Brain magnetic resonance imaging (MRI) was abnormal in 26 patients. Four patients had cerebral atrophy, 14 patients had cerebral and cerebellar atrophy, six patients had cerebellar atrophy, and two patients had brain atrophy with abnormal signals in cerebellar. Brain MRI was normal in 12 patients.

Among the 38 patients, 27 were sporadic, 11 were from families with pedigrees suggestive of either dominant or recessive inheritance. The pedigrees of 11 families were shown in Fig. 1. The clinical details of the 38 patients were shown in Table 1 . Recessively inherited or a de novo heterozygous mutations in established PME genes were identified in 30 patients $(78.9 \%, 30 / 38)$. The gene testing results of the 30 patients were showed in Fig. 2. The genetic causes remained unknown in eight children. All variants and the pathogenic analysis are listed in Table 2.

\section{Mutations in well-recognized PME genes}

Sixteen children had mutations in well-recognized PME genes. Seven genes were identified, including TPP1, PPT1, CLN5, CLN6, CLN7(MFSD8), NEU1 and GBA. One child (P9) was found with one variant in CLN3 inherited from her father.

Pathogenic or likely pathogenic variants in NCLassociated genes were identified in 12 children whose clinical features were consistent with the diagnosis of NCL, including TPP1 in six children, PPT1 in two children, CLN5 in one child, CLN6 in one child, and MFSD 8 (CLN7) in two children.

Six children accepted the test for specific lysosomal enzyme. Five children were found the lack of lysosomal enzyme TPP1 activity, and all of them had mutations in TPP1. Among the five children, one (P6-1) was found with a missense variant (p.Ser538Tyr) and 1 to 3 exons deletion in TTP1.The result was confirmed by Real-time Quantitative PCR (qPCR) (Fig. 3). Enzyme TPP1 activity was also very low in her younger sister (P6-2) who was 


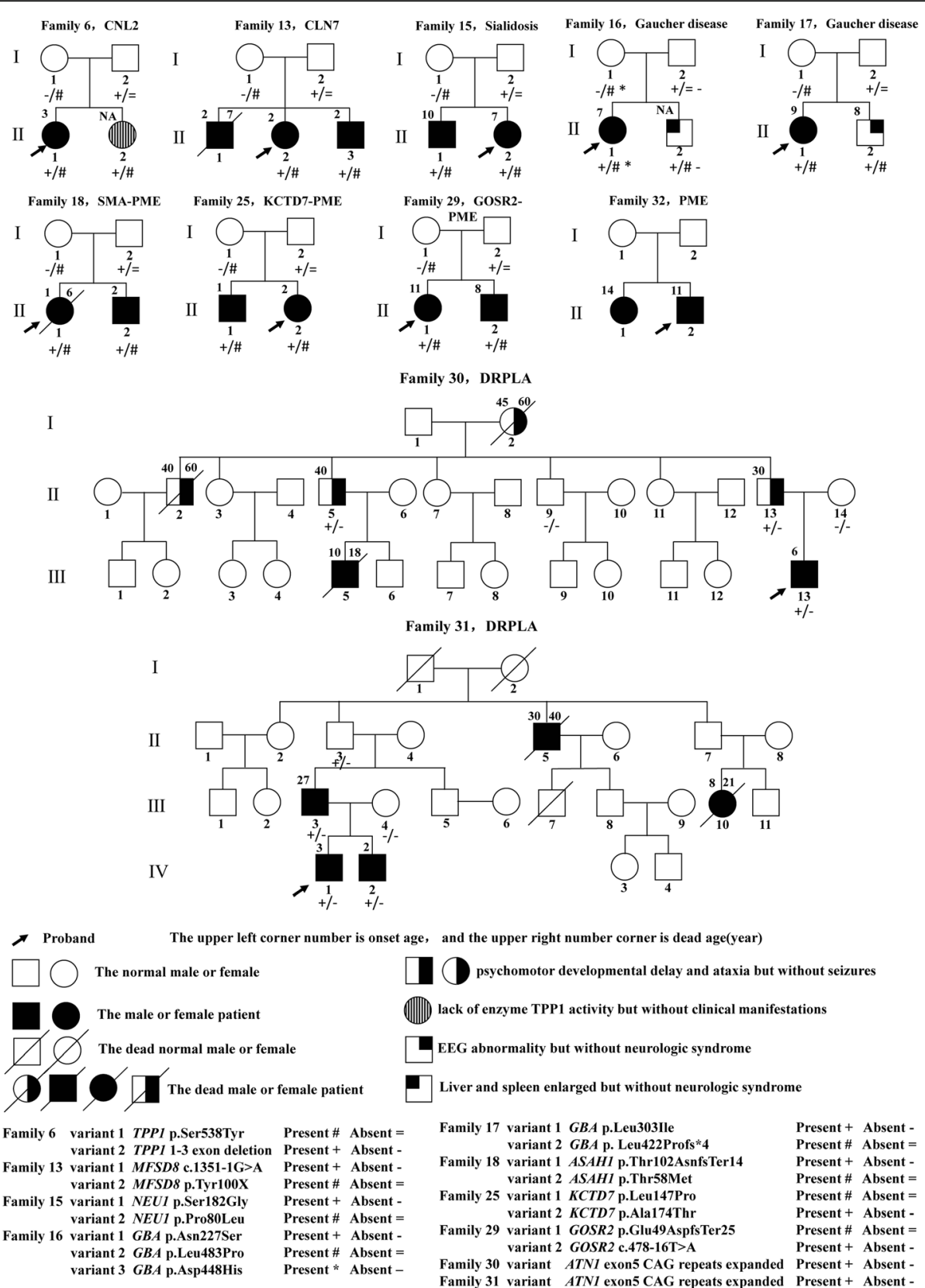

Fig. 1 The 11 family pedigrees of proband and other affected members. SMA-PME: spinal muscular atrophy-progressive myoclonic epilepsy; PME: progressive myoclonic epilepsy; DRPLA: dentatorubral-pallidoluysian atrophy; TPP1: lysosomal enzyme tripeptidy I peptidase; EEG: electroencephalogram

found with the same genotype of P6-1. No clinical manifestations at the last follow-up ( 2 years of age) were observed in P6-2, and further follow-up was needed.

One child (P10) was found PPT1 enzyme deficiency, he was identified with homozygous missense CLN5 mutations (p.Trp151Arg) by trio-based WES. He had seizure onset at the age of 5 years and 7 months, recognition regression was also observed. EEG recordings showed generalized epileptiform discharges, and the brain MRI scans showed cerebellar atrophy.
One child (P9) was found with one variant in CLN3 inherited from her father by trio-based WES. The other variant or Copy number variations (CNVs) had not been found even after reanalyzing the sequencing data carefully. The genetic diagnosis of P9 was not clear. Maybe the whole genome sequencing could be used to find disease-causing genes. She had seizure onset at the age of 2 years and 11 months, the seizure types included myoclonic seizure, focal seizure and generalized tonicclonic seizure (GTCS). Recognition regression was 


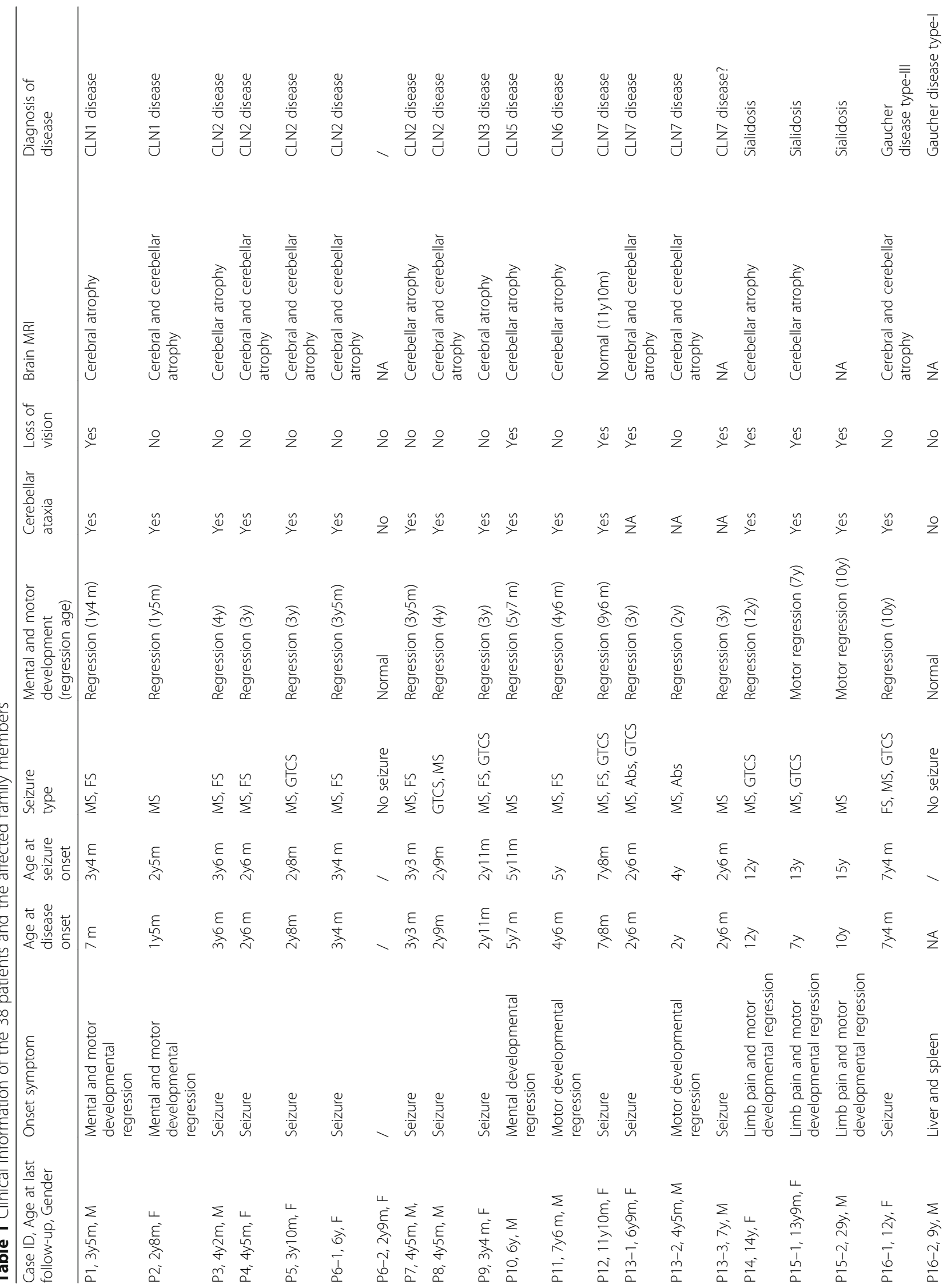




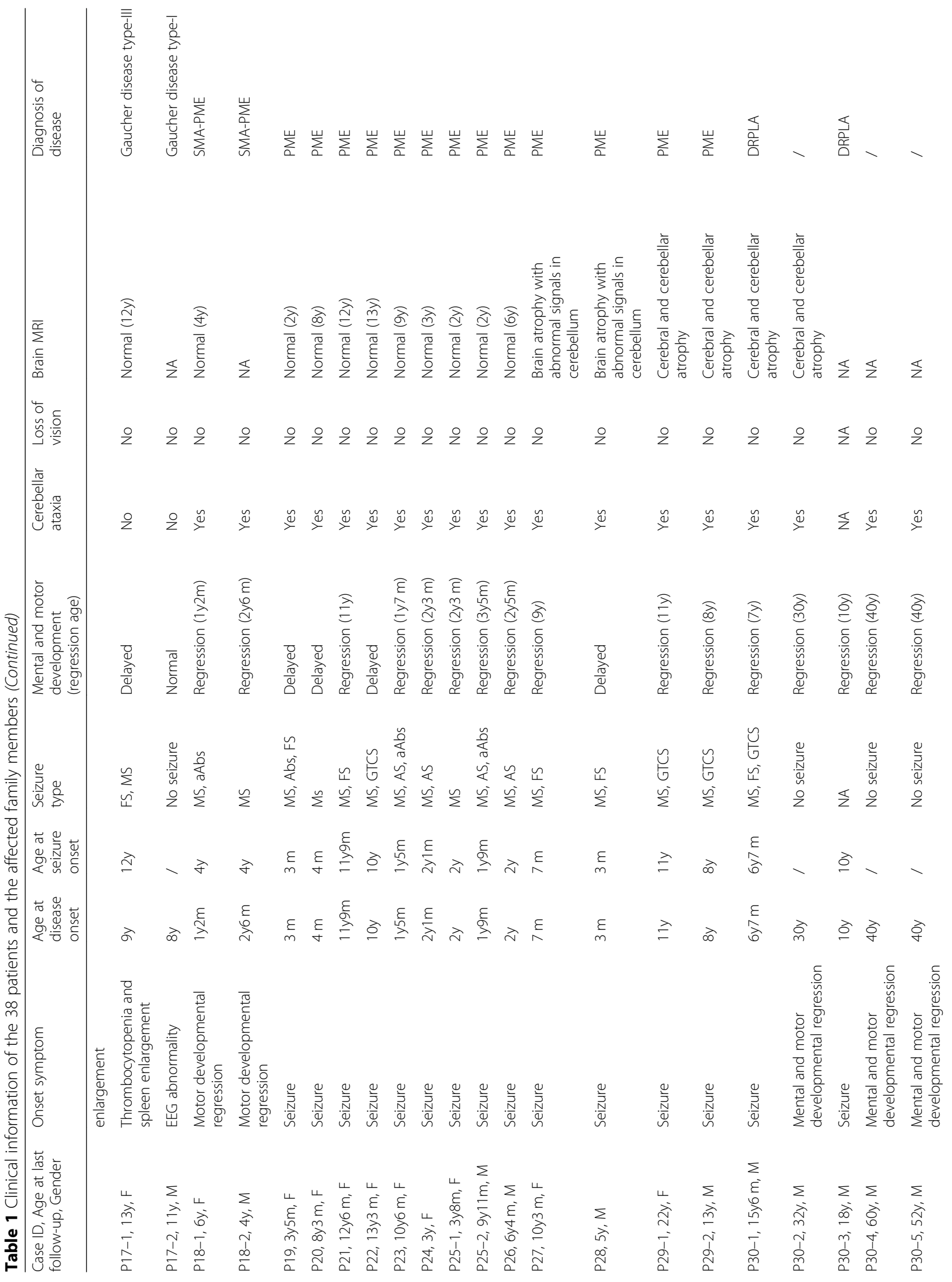


Zhang et al. Acta Epileptologica $\quad$ (2020) 2:14

Page 6 of 15

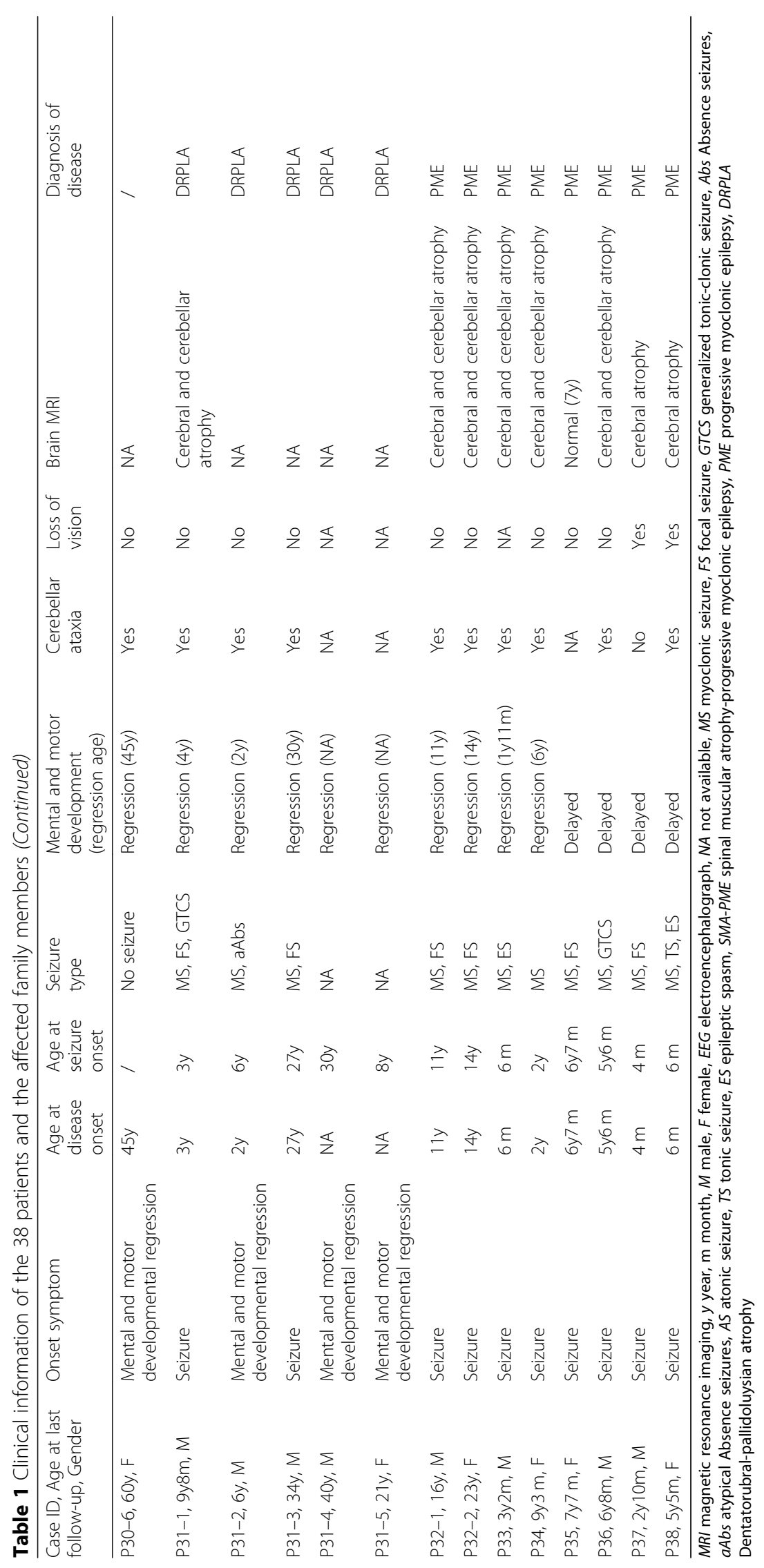




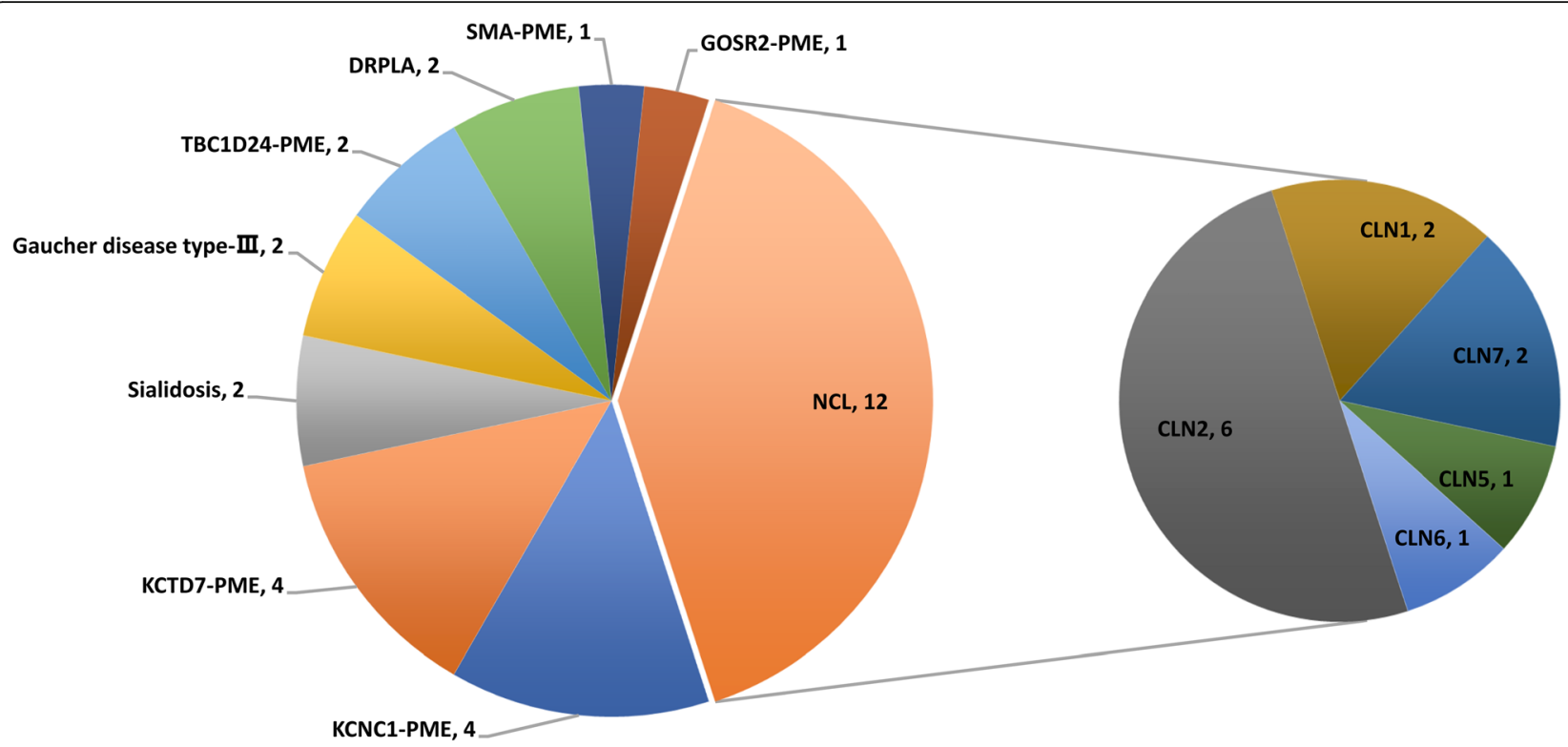

Fig. 2 The gene testing results of 30 patients with established PME genes. PME: progressive myoclonic epilepsy; DRPLA: dentatorubralpallidoluysian atrophy; SMA-PME: spinal muscular atrophy-progressive myoclonic epilepsy; NCL: neuronal ceroid lipofuscinoses

observed at 3 years old. EEG recordings showed generalized epileptiform discharges, and the brain MRI scans showed Cerebral atrophy.

Two unrelated children (P14 and P15-1) had the same compound heterozygous mutations in NEU1 (p.Ser182Gly and p.Pro80Leu). P14 had a cherry red spot in the maculae of both eyes and her genetic testing was conducted by NGS epilepsy panel. Another P15-1 had atrophy of the optic nerve in both eyes, and her elder brother (P15-2) had similar phenotype and the same genotype. According to the clinical manifestation, they were suspected of the diagnosis of sialidosis. Mutations of NEU1 were detected by sanger sequencing. Combining with the clinical phenotype and gene result, they were diagnosed with sialidosis.

Two children (P16-1 and P17-1) had mutations in $G B A$ and deficiency of the lysosomal enzyme glucocerebrosidase, both of them had mental and motor development delay or regression and seizures, and they were diagnosed with neuronopathic Gaucher disease. P16-1 was found with three variants in $G B A$, the variant p.Asn227Ser was paternal, the variants p.Leu483Pro and p.Asp448His were maternal. The three variants are pathogenic or likely pathogenic according to the American College of Medical Genetics and Genomics (ACMG) guidelines [23]. Her younger brother (P16-2) only had liver and spleen enlarged without neurologic manifestations, who had the same three variants in GBA. P16-2 was diagnosed with Gaucher disease type-I. P17-1 had compound heterozygous mutations in GBA, p.Leu303Ile and p.Leu422Profs*4. The younger brother (P17-2) was found with the same mutations in $G B A$, but he only had electroencephalograph abnormality at 8 years old. The last follow age of P17-2 was 11 years old, and he had no seizures or any other clinical symptoms. The further follow-up was needed.

\section{Mutations in newly reported PME-related genes}

Twelve children were found with newly reported PMErelated gene mutations. Five genes were identified, including ASAH1, KCNC1, KCTD7, TBC1D24 and GOSR2.

One patient (P18-1) manifested with severe motor development regression at the age of 1 year and 2 months, followed by frequent myoclonic seizures at the age of 3 years and 9 months. She died of status epilepticus at the age of 6 years. Her younger brother (P18-2) had similar clinical manifestations. The genetic testing by NGS epilepsy panel showed that both of them had compound heterozygous mutations in ASAH1 (p.Thr102AsnfsTer14 and p.Thr58Met). Combined with the phenotype and genotype features, they were diagnosed with spinal muscular atrophy-progressive myoclonic epilepsy (SMAPME).

Four children were found with de novo heterozygous mutations in KCNC1. The genetic testing of three were by trio-based WES, and one by NGS epilepsy panel. Two children (P21 and P22) had the same mutation p.Arg320His in $K C N C 1$, which affected a highly conserved arginine residue in segment S4. This variant was reported to be a recurrent mutation [5, 24]. Two children (P19 and P20) were identified with the mutation p.Ala421Val in KCNC1, which was in the segment S6. What interesting is that we found the phenotypic differences were associated with different genotypes. The 


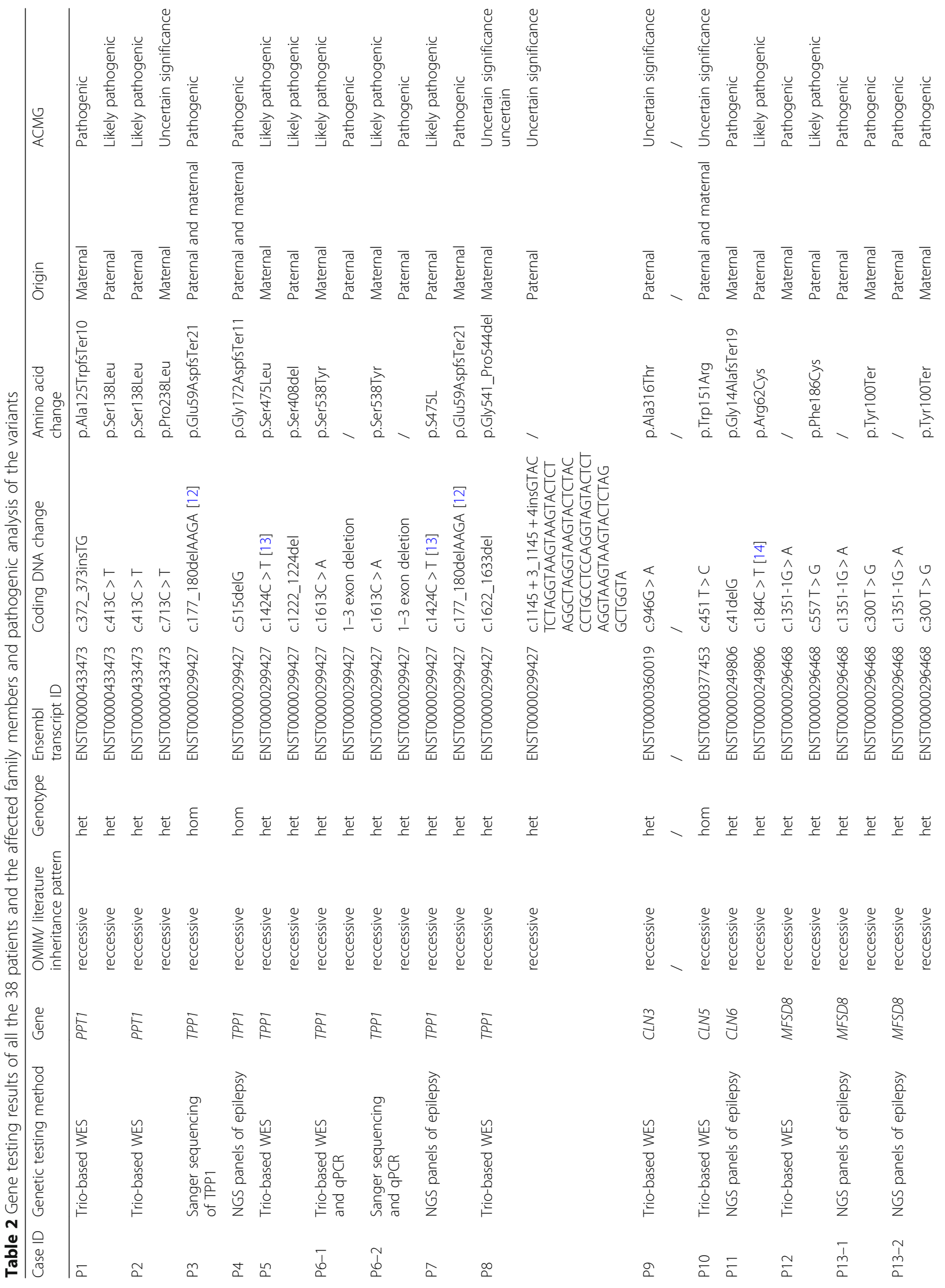




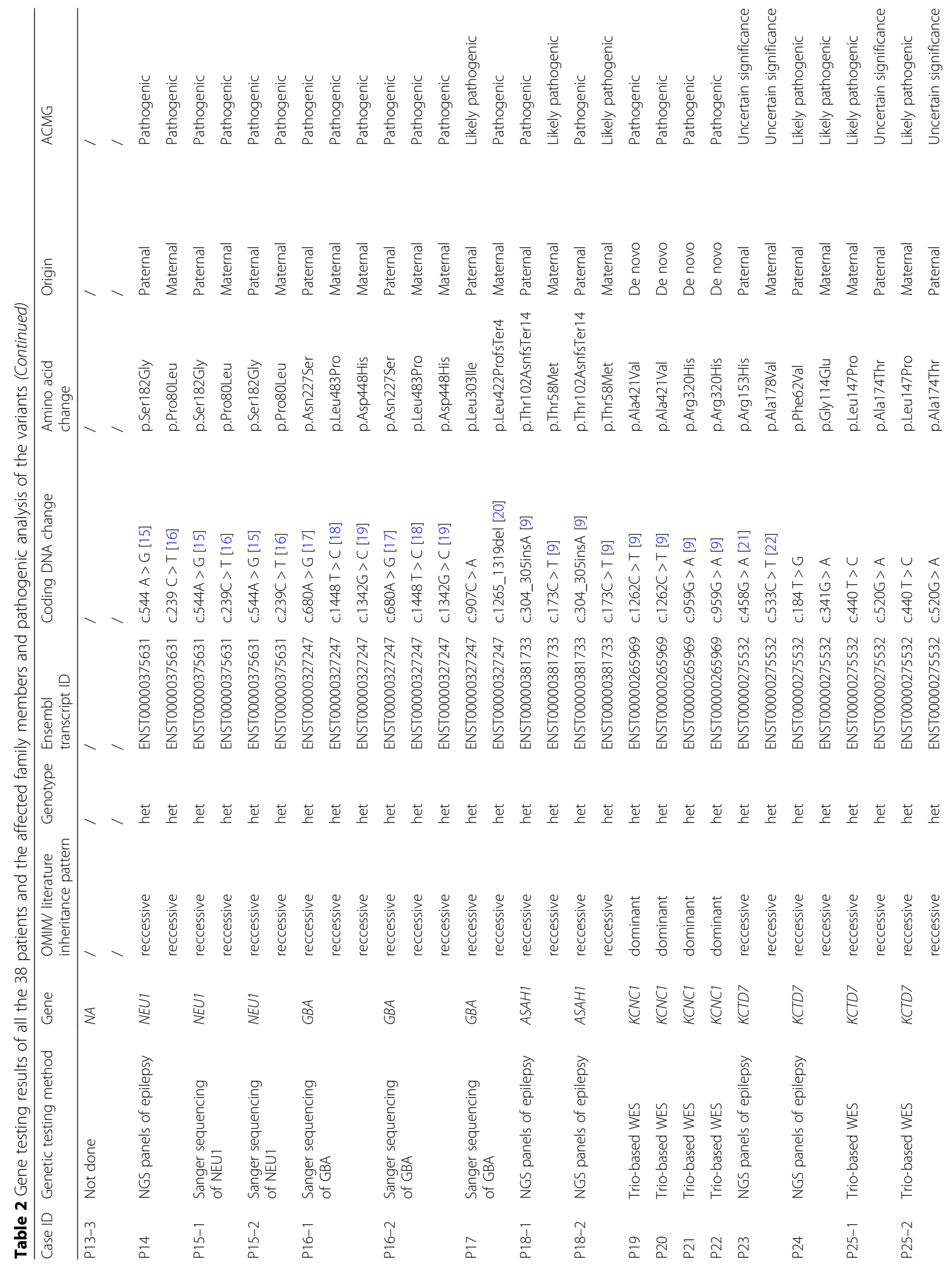


Zhang et al. Acta Epileptologica

(2020) 2:14

Page 10 of 15

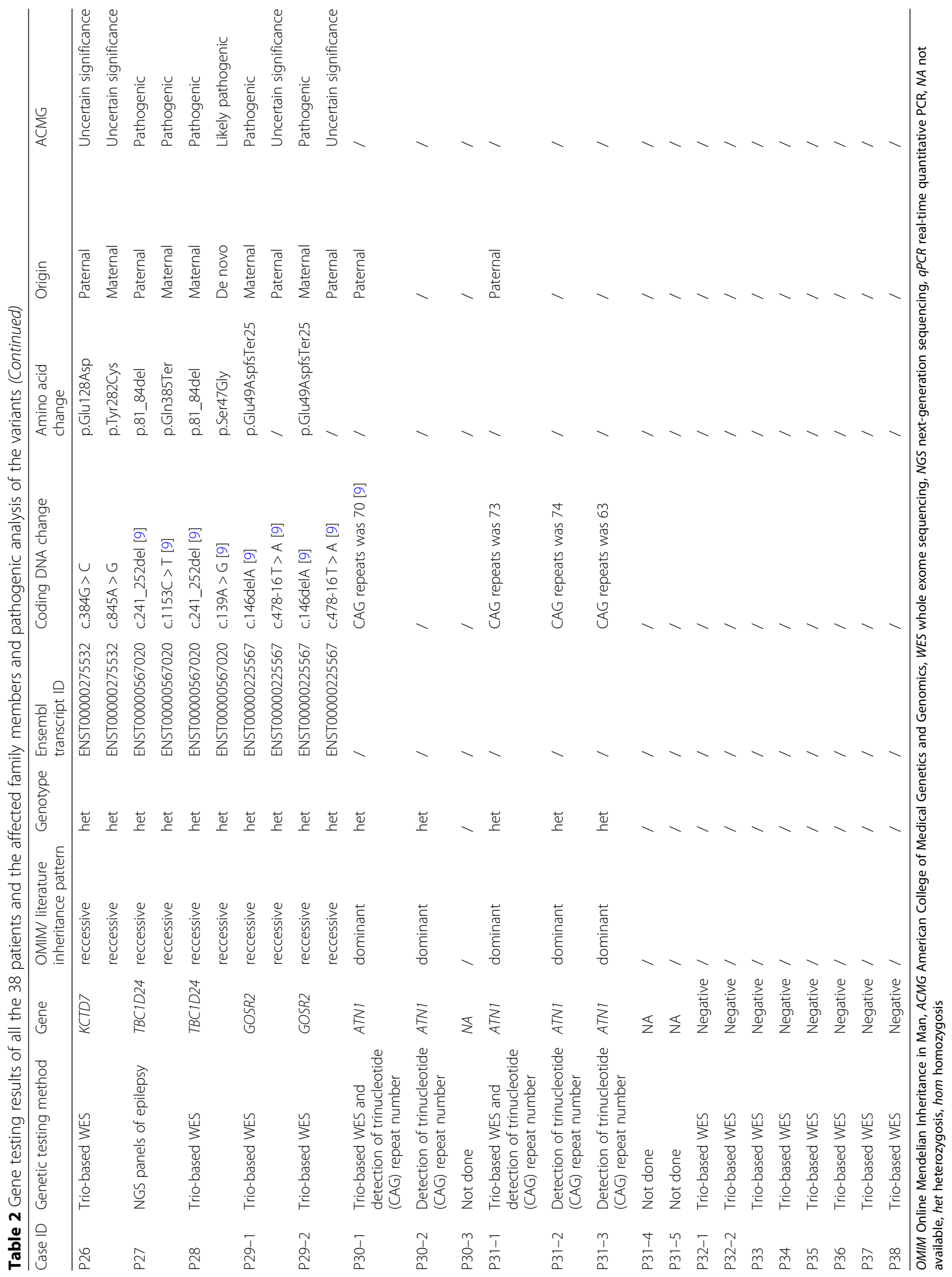




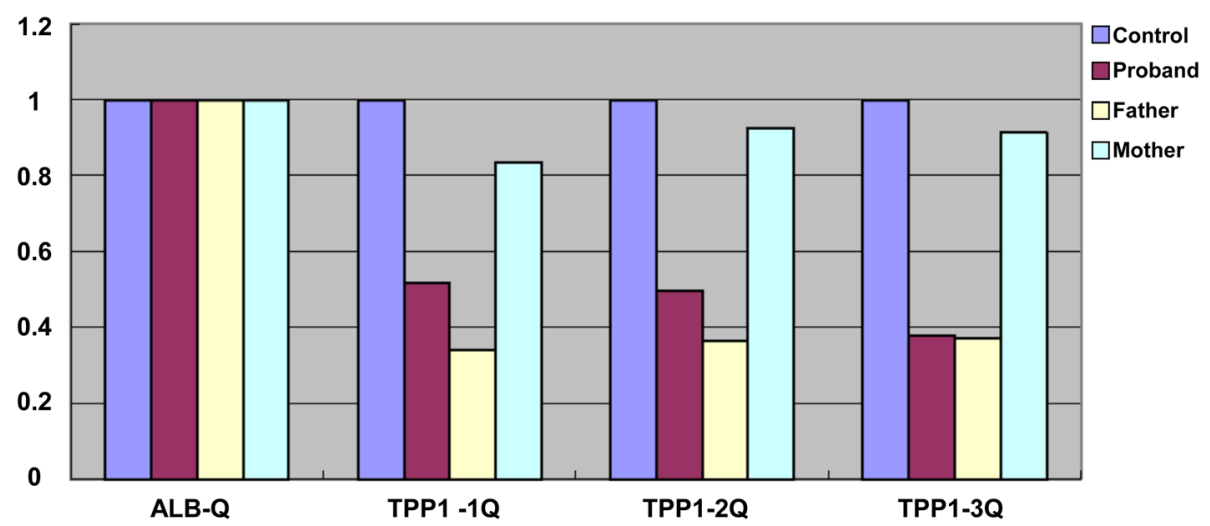

Fig. 3 Real-time quantitative PCR results of patient P6-1 and her parents. The qPCR results confirmed the deletion of exons 1-3 in TPP1 gene in patient P6-1 and her father as compared to a normal control

seizure onset age of two children with mutation p.Ala421Val were 3 and 4 months old respectively, and the seizure onset age of two children with mutation p.Arg320His were 11 years and 9 months and 10 years old respectively. The seizure onset age of children with the variant p.Ala421Val is much earlier than that of with the variant p.Arg320His. MRI scans were normal in four children with KCNC1 mutations, and the last follow-up age was ranged from 3 years and 5 months old to 13 years and 3 months old.

Four children (P23, P24, P25-1 and P26) were found with compound heterozygous mutations in KCTD7 by trio-based WES and NGS panel of epilepsy respectively. Their clinical phenotypes were similar, and the seizure onset age was between 1 year and 5 months to 2 years and 1 month old. Frequent myoclonic seizures and atonic seizures were common, and GTCS were rare. EEG recordings showed generalized epileptiform discharges with large number of discharges in Rolandic area in some cases. MRI scans were normal in four children, and the last follow-up age was ranged from 3 to 10 years and 6 months old. There were eight different variants in four children, and no recurrent variants were found. The elder brother (P25-2) of P25-1 had similar clinical manifestations and the same genotype (p.Leu147Pro and p.Ala174Thr).

Compound heterozygous mutations in TBC1D24 were identified in two children (P27 and P28) by trio-based WES and NGS panel of epilepsy respectively, they were p.81_84del in both children, p.Gln385Ter and p.Ser47Gly in one child each. Two patients exhibited similar clinical phenotypes. The seizure onset age was at 7 and 3 months respectively. The frequent multifocal myoclonus was prominent feature, which could last for several hours to 2 weeks and developed into epilepsia partialis continua (EPC). The EPC could be triggered by fever or infections and could be terminated by sleep or sedation drugs especially by chloral hydrate. The multifocal myoclonus and ictal scalp EEG data lacked clear correlation. Both of the two children were found with cerebral and cerebellar atrophy with abnormal signals in cerebellar, which have been described in our previous publication [25]. P27 was found to have sensorineural deafness at the age of 9 years after a severe EPC which last for 14 days, and her hearing was normal before this EPC.

One child (P29-1) was found with two novel variants in GOSR2 (p.Glu49AspfsTer25 and c.478-16 T > A) by trio-based WES. Her younger brother (P29-2) who had similar clinical phenotype harbored the same variants. The seizure onset age of them was 11 and 8 years old respectively. Seizure types included myoclonic seizures and GTCS. The motor development regression was prominent, and they could hardly walk steadily at the age of 13 and 9 years respectively. Their EEG recordings showed generalized epileptiform discharges. The brain MRI scans showed cerebral and cerebellar atrophy in both children. The frameshift variant p.Glu49AspfsTer25 was pathogenic according to ACMG guidelines. The splice-site variant c.478-16 T > A was uncertain significant according to ACMG guidelines.

\section{Two dentatorubral-pallidoluysian atrophy families}

The patient P30-1 had seizures at the age of 6 years and 7 months, the seizure types included myoclonic seizures, focal seizures and GTCS. He had severe mental retardation and motor development regression. EEG recordings showed generalized epileptiform discharges. The brain MRI scans showed cerebral and cerebellar atrophy. There were six family members affected in family of P30-1. His father (P30-2), two uncles (P30-4 and P30-5) and grandmother (P30-6) were found to walk unsteadily with recognition regression at the age of 30 to 45 years, but they did not had seizures. His cousin (P30- 
3) had seizure attacks at the age of 10 years, and he died in sleep at the age of 18 years, which could be sudden unexpected death in epilepsy (SUDEP). Multiple family members had similar clinical manifestations suggested a genetic cause. However, the trio-based WES did not find any disease related gene mutations. Reanalyzed the family history and clinical phenotype, we observed anticipation, a phenomenon characterized by the earlier age of onset and more severe phenotype in successive generations in this family. In addition, the patient could be diagnosed with PME. Therefore, the patient was diagnosed with DRPLA.

DRPLA is an autosomal dominant neurodegenerative disorder caused by CAG triplet expansion in atrophin 1 (ATN1). Fragment analysis with laser induced fluorescence in capillary electrophoresis was performed for the CAG repeats of ATN1. Expanded CAG repeats of ATN1 were detected in probands and the affected family members. The CAG repeats number of proband (P30-1), his father (P30-2), one of the affected uncles (P30-5) were 70, 63 and 62, respectively. The CAG repeats number of his mother (family 30-II-14) and uncle (family 30-II-9) with normal phenotype were less than 20 .

The family of P31-1 had clinical phenotypes similar to the family of P30-1. Five family members were affected in family of P31-1. The patient P31-1 had seizure onset at the age of 3 years, and he exhibited walking unsteadily with intelligence regression at the age of 8 years. The clinical details of his younger brother (P31-2), his father (P31-3), his grandfather (P31-4) and his aunt (P31-5) were shown in Table 1. Expanded CAG repeats of ATN1 were also detected in proband and the affected family members. The CAG repeats number of proband, his younger brother and his father were 73,74 and 63 respectively, whereas the repeats number of his mother (family 31-III-4) and grandfather (family 31-II-3) with normal phenotype were less than 20 and 56 respectively.

\section{The genetic causes remained unknown in seven children}

The genetic causes remained unknown in seven children. The onset symptoms of the seven children were seizure attack, and the onset age ranged from 4 months to 11 years old. The seizure types included myoclonic seizures in seven children, focal seizures in three children, tonic seizure in one child, epileptic spasm in two children and GTCS in one child. All of the seven children had mental retardation and motor development regression. The EEG recordings showed generalized epileptiform discharges in seven children, focal epileptiform discharges in three children and hypsarrhythmia in two children. The brain MRI scans showed cerebral and cerebellar atrophy in four children, cerebral atrophy in two children. The brain MRI scan was normal in one child when she was 7 years old in the last follow up.
Seven children were all diagnosed with PME according to the clinical features, EEG and MRI results. All seven children received trio-based WES, but none of them were found with disease causing mutations. Metabolic laboratory screening was also performed in seven children, and the results were normal.

\section{Discussion}

PME is a group of neurodegenerative diseases with genetic heterogeneity and phenotypic similarities. It is a challenge to make precise clinical diagnosis of specific forms of PME. However, detailed clinical information, history collection, necessary examination and laboratory test could help to make the final diagnosis. The proper genetical testing method may also assist in making precise etiological diagnosis.

More than 30 genes were reported to be related with PME $[5,10,26]$. It was difficult to sequence all the genes one by one. NGS technology enables massively parallel sequencing of multiple genes, which enables fast and comprehensive genetic analysis [27]. However, NGS does not reliably detect triplet repeat expansions, and CAG repeat number analysis was required when DRPLA was considered as the clinical diagnosis [28]. In this study, sanger sequencing of the target gene, NGS panels of epilepsy, trio-based WES and detection of CAG repeat number were used to investigate the genetic causes. Consequently, $78.9 \%$ (30/38) children reached genetic diagnosis, and 13 genes related to PME were identified in our study. Muona et al. exome-sequenced 84 unrelated PME patients of unknown cause and molecularly solved $31 \%$ cases [5]. The rate of genetic diagnosis of our study was higher than Muona et al., which suggested that various of genetic testing methods should be considered in order to improve the molecular diagnostics of PME.

DRPLA is an autosomal-dominant disorder, caused by unstable expansion of CAG repeats of ATN1 [10]. The expanded repeats are unstable and tend to expand further, which leads to earlier age onset and a more severe phenotype in successive generations. This phenomenon is known as anticipation [28]. Some individuals carried alleles of intermediate repeat length, which are not large enough to cause disease, but are large enough to be prone to further expansion in the next generation [29]. Schols et al. reported that the intermediate repeat ranged from 36 to 49 in DRPLA [28]. In our study, the grandfather (family 31-II-3) of P31-1 had normal clinical phenotype, however, his CAG repeats number in ATN1 was 56, which was larger than 50, and was transmitted to next generation with expanded copies. This suggested that the intermediate repeat could be longer than what it is supposed to be. 
In our study, the child P10 was found lysosomal PPT1 deficiency. Homozygous missense mutations c.451 T > C (p.Trp151Arg) in CLN5 were identified using WES. This phenomenon suggested NCL proteins may share common functions or participate in the same biological pathway or process [30]. There are 13 known proteins of NCL family. Unfortunately, the precise functions of many NCL proteins are still unclear. PPT1/CLN1 is one of the lysosomal enzymes, and CLN5 is a soluble lysosomal protein $[30,31]$. Lyly, et al. demonstrated a close relationship between CLN5 and PPT1/CLN1 proteins by showing the protein interactions between the two and significantly increased expression levels of $C \ln 1$ mRNA in the $C \ln 5-/-$ mouse brain tissue. Their results suggest a possible compensatory role for PPT1 in CLN5 deficiency [32]. Those studies could help to explain the phenomenon in our study. However, in our patient with homozygous missense mutations CLN5, PPT1/CLN1 deficiency existed in peripheral blood, but it was difficult to know for sure whether the expression level of PPT1 decreased in his brain tissue.

In our study, PME related gene mutations were identified in 30 patients, and 12 of which (31.6\%) were found with mutations in NCL related genes (PPT1, TPP1, CLN5, CLN6 and MFSD8). The most prevalent gene was TPP1, which was identified in six $(15.8 \%, 6 / 38)$ children. KCNC1 and KCTD7 mutations were found in four children $(10.5 \%, 4 / 38)$ for each. In the study of Muona et al., 11 unrelated exome-sequenced patients (13\%) were identified with the same recurrent de novo mutation c.959G > A (p.Arg320His) in KCNC1, which accounts for the most in 26 molecularly solved patients [5]. The incidence of KCNC1 in our study was close to that in the study of Muona et al.

Apart from p.Arg320His, we found another recurrent de novo mutation c.1262C > T (p.Ala421Val) in KCNC1 which could also cause PME. Interestingly, the phenotype was related to genotype. The seizure onset age of two children with the mutation p.Ala421Val was 3 and 4 months respectively. Three sporadic children were also reported to harbor the mutation p.Ala421 Val, and the seizure onset age of them were 5 months, 3 weeks and 5 months respectively [33]. However, the seizure onset age of two children with mutation p.Arg320His in our study were 11 years and 9 months of age and 10 years old respectively, and the seizure onset age of 22 children in reported study were 3 to 15 years $[5,24]$. Apparently, the seizure onset age of patients with mutation p.Ala421Val is much earlier than that of patients with mutation p.Arg320His.

The onset age varied in PME patients with different genes. In this study, the onset age is before 3 years old for all children who diagnosed with CLN1 (PPT1), SMA-PME (ASAH1), KCTD7 related PME and TBC1D24 related PME, and also for some children who diagnosed with CLN2
(TPP1), CLN7 (MFSD8), KCNC1 related PME and DRPLA (ATN1). Moreover, the onset age could be earlier than 1 year old for some children who diagnosed with CLN1 (PPT1), KCNC1 related PME and TBC1D24 related PME.

The homozygous missense mutations c.430G $>\mathrm{T}$ (p.Gly144Trp) in GOSR2 was firstly reported in four unrelated PME patients in 2011 [8]. The main clinical features of the GOSR2-associated PME are early-onset ataxia, action myoclonus and seizures, relative preservation of cognitive function until the late stages of the disease. GOSR2-associated PME is a rare disease with very few cases reported so far [34, 35]. Most PME patients are homozygous for a p.Gly144Trp mutation and develop similar clinical presentations. Recently, more variants including c.491_493delAGA (p.Lys164del) and c.491_493delAGA (p.K164del) have been reported [36, 37]. The syndrome was called 'North Sea PME' given the fact that all patients originated from countries surrounding the North Sea [34]. It is the first time for GOSR2-associated PME reported in countries outside the area, and both of c.146delA (p.Glu49AspfsTer25) and c.478$16 \mathrm{~T}>\mathrm{A}$ are novel variants. However, the pathogenicity of splice-site variant c.478-16 T > A remains uncertain, and further study such as qPCR is needed for functional analysis. It was the first time for GOSR2 mutations related PME reported in Asia. Our study contributed to expand the genotype of this condition.

The genetic causes remained unknown in eight children. Their clinical features meet the diagnostic criteria of PME. All eight children received trio-based WES, but none of them were found with disease causing mutations. All of the parents of the eight children did not have mental and/or motor development delay or regression or epilepsy, and the CAG triple expansion were not tested in them, which often be test when much family members were affected such as DRPLA. Maybe the whole genome sequencing could be used to find diseasecausing genes.

\section{Conclusion}

The seizure onset age of PME varies, it could be ranged from infant to adult. PME is a group of neurogenetic diseases with phenotypic and genotypic heterogeneity. Accurate diagnosis is very challenging. In combination of detailed clinical phenotype information, specific laboratory results and various of genetic testing methods including sanger sequencing of single gene, NGS panels of epilepsy, trio-based WES and detection of CAG repeat number, etiological diagnosis could finally be obtained. The NCL was most common in PME related diseases. The novel mutations in GOSR2 expanded the genotype of PME, and this gene related PME are not only found in areas surrounding the North Sea. The onset age could be before 3 years old for some children whose diagnosis are CLN1, 
CLN2, CLN7, SMA-PME, KCNC1 related PME, KCTD7 related PME, TBC1D24 related PME, and DRPLA.

\section{Supplementary information}

Supplementary information accompanies this paper at https://doi.org/10. 1186/s42494-020-00023-z.

Additional file 1: Supplementary Table 1. Panel of 535 genes associated with epilepsy.

\section{Abbreviations}

ACMG: American College of Medical Genetics and Genomics; ATN1: Atrophin 1; by CAG: Cytosine-adenine-guanine; CNVs: Copy number variations; DRPLA: Dentatorubral-pallidoluysian atrophy; EEG: Electroencephalogram; EPC: Epilepsia partialis continua; GTCS: Generalized tonic-clonie seizure; MRI: Magnetic resonance imaging; NCL: Neuronal ceroid lipofuscinoses: NGS: Next generation sequencing; PME: Progressive myoclonic epilepsy; PPT1: Lysosomal palmitoyl protein thioesterase; qPCR: Real-time Quantitative PCR; SMA-PME: Spinal muscular atrophy-progressive myoclonic epilepsy; SUDEP: Sudden unexpected death in epilepsy; TPP1: Lysosomal enzyme tripeptidyl peptidase; WES: Whole exome sequencing

\section{Acknowledgments}

We would like to extend our deepest appreciation to the patients and their families participating in this study. We would also like to thank team staff who assisted in the data collection as well as Dr. Xiaodong Wang from Cipher Gene, LLC for her assistance in editing the manuscript.

\section{Authors' contributions}

Jing Zhang is the first author of this manuscript, and she participated in data collection, patients' follow-up, drafting and revising the paper. Ying Yang, Xueyang Niu, Jiaoyang Chen, Wei Sun, Changhong Ding, Lifang Dai, Liping Zhang, Qi Zeng, Yi Chen, Xiaojuan Tian, Xiaoling Yang, Taoyun Ji, Zhixian Yang, Yanling Yang, Yuwu Jiang have made great assistants in data collection and analysis. Professor Yuehua Zhang have made great contribution to study designing, and she is the Corresponding author. All authors read and approved the final manuscript.

\section{Funding}

This work was supported by Key Research Project of the Ministry of Science and Technology of China (grant numbers 2016 YFC0904400 and 2016YFC0904401); The capital health research and development of special (grant number 2016-1-2011).

\section{Availability of data and materials}

The datasets during and/or analysed during the current study available from the corresponding author on reasonable request.

\section{Ethics approval and consent to participate}

This study was approved by the Ethics Committee of Peking University First Hospital. Parental written informed consent was obtained for all children enrolled in this study.

\section{Consent for publication}

Not applicable.

\section{Competing interests}

The authors declare that they have no competing interests.

\section{Author details}

${ }^{1}$ Department of Pediatrics, Peking University First Hospital, No. 1 of Xian Men Street, Xicheng District, Beijing 100034, China. ${ }^{2}$ Department of Neurology, Xuanwu Hospital, Capital Medical University, No. 45 of Changchun Street, Xicheng District, Beijing 100034, China. ${ }^{3}$ Department of Neurology, Beijing Children's Hospital, Capital Medical University, No. 56 of South Lishi Road, Xicheng District, Beijing 100045, China. ${ }^{4}$ Department of Pediatrics, Xuanwu Hospital, Capital Medical University, No. 45 of Changchun Street, Xicheng District, Beijing 100034, China.
Received: 13 April 2020 Accepted: 4 August 2020

Published online: 02 September 2020

\section{References}

1. Proposal for Revised Classification of Epilepsies and Epileptic Syndromes. Commission on classification and terminology of the International League Against Epilepsy. Epilepsia. 1989;30(4):389-99.

2. Genetics of epilepsy and genetic epilepsies., John Libbey Eurotext, France, 2009.

3. Minassian BA. The progressive myoclonus epilepsies. Prog Brain Res. 2014; 213:113-22.

4. Hallmann K, Zsurka G, Moskau-Hartmann S, Kirschner J, Korinthenberg R, Ruppert AK, et al. A homozygous splice-site mutation in CARS2 is associated with progressive myoclonic epilepsy. Neurology. 2014;83(23):2183-7.

5. Muona M, Berkovic S, Dibbens L, Oliver K, Maljevic S, Bayly M, et al. A recurrent de novo mutation in $\mathrm{KCNC} 1$ causes progressive myoclonus epilepsy. Nat Genet. 2015;47(1):39-46.

6. Van Bogaert P. KCTD7-related progressive myoclonus epilepsy. Epileptic Disord. 2016;18(S2):115-9.

7. Siintola E, Topcu M, Aula N, Lohi H, Minassian BA, Paterson AD, et al. The novel neuronal ceroid lipofuscinosis gene MFSD8 encodes a putative lysosomal transporter. Am J Hum Genet. 2007;81(1):136-46.

8. Corbett MA, Schwake M, Bahlo M, Dibbens LM, Lin M, Gandolfo LC, et al. A mutation in the Golgi Qb-SNARE gene GOSR2 causes progressive myoclonus epilepsy with early ataxia. Am J Hum Genet. 2011;88(5):657-63.

9. Zhang J, Zhang YH, Chen JY, Ji TY, Yang ZX, Yang XL, et al. Pathogenic gene variants and clinical phenotype features of 26 children with progressive myoclonic epilepsy. Zhonghua Er Ke Za Zhi. 2019;57(6):458-64

10. Shahwan A, Farrell M, Delanty N. Progressive myoclonic epilepsies: a review of genetic and therapeutic aspects. Lancet Neurol. 2005:4(4):239-48.

11. Michelucci R, Pasini E, Riguzzi P, Andermann E, Kälviäinen $R$, Genton P. Myoclonus and seizures in progressive myoclonus epilepsies: pharmacology and therapeutic trials. Epileptic Disord. 2016:18:145-53.

12. Chang $X$, Huang $Y$, Meng $H$, Jiang $Y, W u Y$, Xiong $H$, et al. Clinical study in Chinese patients with late-infantile form neuronal ceroid lipofuscinoses. Brain Dev. 2012:34(9):739-45.

13. Sleat DE, Gin RM, Sohar I, Wisniewski K, Sklower-Brooks S, Pullarkat RK, et al. Mutational analysis of the defective protease in classic late-infantile neuronal ceroid lipofuscinosis, a neurodegenerative lysosomal storage disorder. Am J Hum Genet. 1999;64(6):1511-23.

14. Cannelli N, Garavaglia B, Simonati A, Aiello C, Barzaghi C, Pezzini F, et al. Variant late infantile ceroid lipofuscinoses associated with novel mutations in CLN6. Biochem Biophys Res Commun. 2009;379(4):892-7.

15. Chen CM, Lai SC, Chen IC, Hsu KC, Lyu RK, Ro LS, et al. First report of two Taiwanese siblings with sialidosis type I: a 10-year follow-up study. J Neurol Sci. 2006:247(1):65-9.

16. Itoh K, Naganawa Y, Matsuzawa F, Aikawa S, Doi H, Sasagasako N, et al. Novel missense mutations in the human lysosomal sialidase gene in sialidosis patients and prediction of structural alterations of mutant enzymes. J Hum Genet. 2002:47(1):29-37.

17. Montfort M, Chabás A, Vilageliu L, Grinberg D. Functional analysis of 13 GBA mutant alleles identified in Gaucher disease patients: pathogenic changes and "modifier" polymorphisms. Hum Mutat. 2004:23(6):567-75.

18. Tammachote $\mathrm{R}$, Tongkobpetch S, Srichomthong C, Phipatthanananti K, Pungkanon S, Wattanasirichaigoon D, et al. A common and two novel GBA mutations in Thai patients with Gaucher disease. J Hum Genet. 2013;58(9): $594-9$

19. Liou B, Kazimierczuk A, Zhang M, Scott CR, Hegde RS, Grabowski GA. Analyses of variant acid beta-glucosidases: effects of Gaucher disease mutations. J Biol Chem. 2006;281(7):4242-53.

20. Beutler E. Gaucher disease as a paradigm of current issues regarding single gene mutations of humans. Proc Natl Acad Sci U S A. 1993;90(12):5384-90.

21. Nykamp K, Anderson M, Powers M, Garcia J, Herrera B, Ho YY, et al. Sherloc: a comprehensive refinement of the ACMG-AMP variant classification criteria. Genet Med. 2017;19(10):1105-17.

22. Mastrangelo M, Sartori S, Simonati A, Brinciotti M, Moro F, Nosadini M, et al. Progressive myoclonus epilepsy and ceroidolipofuscinosis 14: The multifaceted phenotypic spectrum of KCTD7-related disorders. Eur J Med Genet. 2019;62(12):103591.

23. Richards S, Aziz N, Bale S, Bick D, Das S, Gastier-Foster J, et al. Standards and guidelines for the interpretation of sequence variants: a joint consensus 
recommendation of the American College of Medical Genetics and Genomics and the Association for Molecular Pathology. Genet Med. 2015; 17(5):405-24.

24. Oliver KL, Franceschetti S, Milligan CJ, Muona M, Mandelstam SA, Canafoglia $\mathrm{L}$, et al. Myoclonus epilepsy and ataxia due to KCNC1 mutation: analysis of 20 cases and $K(+)$ channel properties. Ann Neurol. 2017;81(5):677-89.

25. Zhang J, Chen J, Zeng Q, Zhang L, Tian X, Yang X, et al. Infantile epilepsy with multifocal myoclonus caused by TBC1D24 mutations. Seizure. 2019;69: 228-34.

26. Girard JM, Turnbull J, Ramachandran N, Minassian BA. Progressive myoclonus epilepsy. Handb Clin Neurol. 2013;113:1731-6.

27. Wright $C F$, Fitzpatrick DR, Firth HV. Paediatric genomics: diagnosing rare disease in children. Nat Rev Genet. 2018;19(5):253-68.

28. Schols L, Bauer P, Schmidt T, Schulte T, Riess O. Autosomal dominant cerebellar ataxias: clinical features, genetics, and pathogenesis. Lancet Neurol. 2004;3(5):291-304.

29. Soong BW, Paulson HL. Spinocerebellar ataxias: an update. Curr Opin Neurol. 2007;20(4):438-46.

30. Huber RJ. Using the social amoeba Dictyostelium to study the functions of proteins linked to neuronal ceroid lipofuscinosis. J Biomed Sci. 2016;23(1):83.

31. Kousi M, Lehesjoki AE, Mole SE. Update of the mutation spectrum and clinical correlations of over 360 mutations in eight genes that underlie the neuronal ceroid lipofuscinoses. Hum Mutat. 2012;33(1):42-63.

32. Lyly A, Von Schantz C, Heine C, Schmiedt ML, Sipila T, Jalanko A, et al. Novel interactions of CLN5 support molecular networking between neuronal Ceroid Lipofuscinosis proteins. BMC Cell Biol. 2009:10:83.

33. Park J, Koko M, Hedrich UBS, Hermann A, Cremer K, Haberlandt E, et al. KCNC1-related disorders: new de novo variants expand the phenotypic spectrum. Ann Clin Transl Neurol. 2019;6(7):1319-26.

34. Boisse Lomax L, Bayly MA, Hjalgrim H, Moller RS, Vlaar AM, Aaberg KM, et al. 'North Sea' progressive myoclonus epilepsy: phenotype of subjects with GOSR2 mutation. Brain. 2013;136(Pt 4):1146-54.

35. Dibbens LM, Rubboli G. GOSR2: a progressive myoclonus epilepsy gene. Epileptic Disord. 2016;18(S2):111-4.

36. Praschberger R, Balint B, Mencacci NE, Hersheson J, Rubio-Agusti I, Kullmann $\mathrm{DM}$, et al. Expanding the phenotype and genetic defects associated with the GOSR2 Gene. Mov Disord Clin Pract. 2015:2(3):271-3.

37. Volker JM, Dergai M, Abriata LA, Mingard Y, Ysselstein D, Krainc D, et al. Functional assays for the assessment of the pathogenicity of variants of GOSR2, an ER-to-Golgi SNARE involved in progressive myoclonus epilepsies. Dis Model Mech. 2017;10(12):1391-8.

Ready to submit your research? Choose BMC and benefit from:

- fast, convenient online submission

- thorough peer review by experienced researchers in your field

- rapid publication on acceptance

- support for research data, including large and complex data types

- gold Open Access which fosters wider collaboration and increased citations

- maximum visibility for your research: over $100 \mathrm{M}$ website views per year

At $\mathrm{BMC}$, research is always in progress.

Learn more biomedcentral.com/submissions 